Acta Crystallographica Section A

Foundations of Crystallography

ISSN 0108-7673

Received 8 June 2012

Accepted 9 July 2012

\section{Background to the Nobel Prize to the Braggs}

\author{
Anders Liljas \\ Biochemistry and Structural Biology, Lund University, Lund, Sweden. Correspondence e-mail: \\ anders@|iljas.net
}

\begin{abstract}
The Nobel Committees have to follow the nominations submitted for a specific year. During the early phase of X-ray crystallography, a limited number of scientists were active. In 1914 Max von Laue and William Henry Bragg were both nominated and could have been awarded a joint Nobel Prize. However, a member of the Nobel Committee for Physics, Allvar Gullstrand, was well aware of the activities in the field and strongly recommended that only von Laue should receive the prize since a main contributor, William Laurence Bragg, was not nominated. Next year, when the First World War had started, there were few nominations, but now both Braggs, father and son, were nominated. Gullstrand was very pleased and recommended them both for the 1915 Nobel Prize in Physics. The rest of the committee agreed and this then became the decision of the Royal Academy for Sciences, Stockholm.
\end{abstract}

The will of Alfred Nobel states that the prizes shall be awarded in the fields of physics, chemistry, physiology or medicine, literature and peace. The organizations which are to award the prizes are also stated. In the case of physics and chemistry the Royal Academy of Sciences, Stockholm, was given the task of deciding on these. Here the classes of physics and chemistry elect a five-member committee, possibly with some adjunct members, who will work with nominations received from all over the world in order to recommend to the Academy a recipient for the prize. The invited nominators in a particular year are expected to reply to the relevant committee before 1 February. To be awarded a prize, a scientist has to have been nominated in that specific year. The relevant Nobel Committee performs extensive work to identify the strongest candidate or candidates (maximally three) from the nominations for that specific year. Many of the nominees are thoroughly evaluated by members of the committee or knowledgeable scientists. The relevant class of the Academy (chemistry or physics) subsequently discusses the proposal by the Nobel Committee and gives their recommendations to the Academy, where the final voting is done. The Royal Academy of Sciences in Stockholm safely stores all of the protocols of the Academy and its Nobel Committees. In the case of the oldest protocols they are all written in a clear and legible hand.

In the protocols from the Nobel Committee for Physics for 1914 one finds that 44 nominations were made for the Nobel Prize and 24 scientists were nominated (some further details are presented by Eckert, 2012). Among the nominators was Svante Arrhenius (Stockholm, a member of the Academy and Swedish Nobel Prize winner in chemistry 1903 for his electrolytic theory of dissociation) who nominated William Henry Bragg (Leeds) together with Henry Gwyn Jeffreys Moseley (Manchester and Oxford) and Charles Galton
Darwin (Manchester) for their studies of the periodic system using various elements as anticathodes for generating X-rays. At the time, quite a number of scientists were engaged in exploring the nature and use of X-rays. It was well known, through the observations by Charles Glover Barkla (Nobel Prize in Physics 1917, but not nominated 1914 or 1915), that when X-rays hit some material secondary (fluorescent) X-rays emerge. These secondary X-rays are characteristic of the material being irradiated. He also could conclude that the hardness (wavelength) of this secondary radiation was related not to the atomic weight, but to the atomic number. Professor Emil Warburg (Charlottenburg) nominated Max von Laue (Munich) jointly with W. H. Bragg (Leeds). Professor Adolf von Baeyer (Munich) nominated von Laue alone. Allvar Gullstrand (Uppsala) was given the task of evaluating the work of von Laue. Gullstrand was an MD who specialized in ophthalmology and a Nobel laureate in physiology or medicine for 1911. His primary expertise was in geometrical and physiological optics. He was a member of the Academy and the Nobel Committee for Physics from 1911 to 1929 and was the chairman of the committee from 1922 to 1929 (see http:// www.nobelprize.org).

The evaluation by Gullstrand (3 July 1914) has the title (my translation from Swedish): 'Evaluation concerning the discovery by von Laue of the bending of X-rays in crystal lattices and the usage by W. H. Bragg of this phenomenon for research on crystal structures'. The report discusses attempts to identify the nature of X-rays. In particular, the failure to bend X-rays by strong magnets suggested that they could not be charged particles. W. H. Bragg, who had been publishing on the topic since 1908, was of the opinion that X-rays could be particles composed of two neutralizing charges such as a positive alpha particle (then not known to be doubly charged) and a negatively charged electron. Furthermore, it was 
observed that by passing X-rays from one medium to another the direction of the beam was not changed. Thus, if the $\mathrm{X}$-rays were a wave phenomenon, like light, the wavelengths must be very small.

Gullstrand continues on to say that this was the state of understanding of the nature of X-rays when von Laue suggested irradiating a crystal and studying the consequent effects. If X-rays had wavelengths of a similar magnitude to the lattice spacings in crystals, interference would occur. von Laue worked out a theory for the experiment that predicted what result would be expected. W. Friedrich and P. Knipping performed the simple experiment on several different types of crystals. von Laue reported the successful results together with the theory in the session documents of the Munich Academy 1912 (Friedrich et al., 1912; von Laue, 1912). This epochmaking discovery of the diffraction of X-rays by crystals gave science a new tool of great significance and at the same time allowed von Laue to conclude that X-rays were a wave phenomenon. The wavelengths were evidently of the same range as the distances between atoms in the crystal. The possibility that the observations were due to X-ray fluorescence was easily disproven. However, some of the expected maxima of blackening of the photographic plates from a zincblende (sphalerite) crystal did not appear. von Laue assumed that this could be due to several cubic lattices displaced from each other in the crystal (von Laue, 1912).

Gullstrand further discusses letters to Nature by W. H. Bragg concerning the crystal interferences observed by von Laue. Bragg suggested that 'avenues' in the crystal could guide the X-rays in directions different from the initial one (W. H. Bragg, 1912). He also claimed that his son, William Lawrence Bragg, had confirmed his views in a presentation to the Cambridge Philosophical Society. However, in the written form of the presentation (W. L. Bragg, 1913a) Gullstrand finds no support of the idea of avenues but rather a new theory, where the dark spots on the photographic plate could be considered as reflections of the X-ray beam by planes in the crystal lattice. The theory of Bragg was a simple way to express the theory developed by von Laue.

In June 1913 the two Braggs submitted separate notes to the Royal Society, where the younger Bragg further describes the reflection aspect of diffraction (W. L. Bragg, 1913b). Planes that are richly populated by atoms will give more prominent diffraction spots. The possibility to determine the atomic arrangement from the spots and their intensities then becomes a natural consequence. W. L. Bragg in this way had developed means by which he could determine crystal structures. He then used these methods to determine the structures of crystals of alkali metal halides, and subsequently the structures of diamond and zincblende and a range of other types of crystals. At the same time, W. H. Bragg investigated the spectra produced by a range of anticathode elements (W. H. Bragg, 1913b).

It is remarkable that Gullstrand thoroughly discusses observations by W. L. Bragg who was not nominated for the prize in 1914. This is probably partly due to the joint nomination of von Laue and W. H. Bragg by Professor E. Warburg.
This could have resulted in the Academy jointly awarding the prize to these two as an appropriate way to deal with two closely related discoveries. Gullstrand was very much against such a decision since it would miss a very central contributor, W. L. Bragg. Rather Gullstrand's conclusion was, along with the nomination by Professor von Baeyer, to recommend awarding the prize to von Laue alone. Gullstrand, with significant pleasure, also comments on the short time between the time of the discovery and the time of a possible award as well as the great value for mankind that X-ray diffraction analysis will have. Rarely would the Academy have the opportunity to find a laureate in better agreement with the wording of the will of Alfred Nobel.

The Nobel Committee for Physics (Gustaf Granquist, Allvar Gullstrand, B. Hasselberg, V. Carlheim-Gyllensköld and Svante Arrhenius) on 15 September 1914 summarized their conclusions concerning the nominations for 1914. The committee mentions the joint nomination of W. H. Bragg and von Laue, but also highlights the contributions by W. L. Bragg, even though he was not nominated in that year. The committee concluded that through these discoveries 'a new means has been generated for the studies of the inner construction of crystals, with immense consequences, no less for the chemical than for the mineralogical sciences'. Their recommendation to the Academy was that the Nobel Prize in Physics 1914 should be awarded to Max von Laue 'for his discovery of the diffraction of X-rays in crystals'. The physics class of the Academy agreed with this recommendation at their meeting on 31 October 1914.

However, 1914 became a year of great problems due to the outbreak of the First World War. This also affected scientific activities and the work of scientific academies. For this reason, the Royal Academy requested in a letter dated 16 October 1914 to the Board of the Nobel Foundation a wish to discuss whether the prizes for the year should be delayed. The Board of the Nobel Foundation, together with members of the awarding institutions, convened on 20 October and suggested that, if the awarding institutions all were of the same opinion, permission should be requested from the Swedish government to award the Nobel Prizes for 1914 together with the prizes for 1915 on 1 June 1916. Presumably they expected that the war would be over by then. It was also explicitly stated that the recipients should benefit from the additional interest on their prize money due to this delay.

The Swedish government decided that the Nobel Prizes for 1914 could be awarded in 1915, but not delayed until 1916. Therefore, on 28 October 1914 the Academy postponed the decision on the award of the prizes for 1914. The Nobel Prizes for 1914 were reserved to be awarded in 1915. This created some confusion among nominating scientists. Since no winner had been announced for 1914, Max von Laue was again nominated for the prize in 1915.

In 1915 there were only 17 nominators for the prize in physics (compared to 1914 when there were 44) and 21 scientists were nominated (in 1914 there were 24). T. W. Richards (Cambridge, MA) nominated both W. H. and W. L. Bragg. H. A. Bumstead (New Haven) nominated Max von 
Laue alone or jointly with the two Braggs. Stefan Meyer (Vienna) nominated W. H. Bragg together with Max von Laue. Furthermore, Svante Arrhenius again nominated W. H. Bragg, now in both physics and chemistry, as before together with Henry Gwyn Jeffreys Moseley and Charles Galton Darwin (Arrhenius, 1915).

The Nobel Committee again gave Allvar Gullstrand the task of reviewing the work of W. H. Bragg, now together with his son W. L. Bragg. Since the Academy had not yet decided about the prize for 1914, it was Gullstrand's opinion that if von Laue was not awarded in 1914 he should get the prize for 1915.

Gullstrand's report from 25 June 1915 has the title (my translation): 'Evaluation concerning the investigations by $\mathrm{W}$. H. Bragg and W. L. Bragg of crystal structures and X-ray radiation'. He reminds the readers that the possibility of rewarding von Laue together with W. H. Bragg had already been discussed a year ago. He makes it clear that this would have been a mistake, since the contributions of the younger Bragg were highly significant and he was not nominated for the prize in 1914. Gullstrand further states that in the work on crystal structures W. L. Bragg had made the most significant contributions. The father and son published their findings in a rapid flow of scientific reports and also in a book, $X$-rays and Crystal Structure (Bragg \& Bragg, 1915).

Gullstrand states that when von Laue initially made his discovery and the first applications were made, two theories got a severe blow. One was the view, supported by W. H. Bragg, that X-rays were a radiation of particle nature. The second concerned the zincblende structure, where von Laue had assumed a primitive lattice. According to Barlow and Pope face-centred lattices would give the densest packing of balls. W. L. Bragg, in his first report on the bending of X-rays and his interpretation of the zincblende structure, finds it to be face centred (W. L. Bragg, 1913a) and thanks Professor Pope for advice. The same paper contains the outlines of methods by which the discovery by von Laue can become useful for crystallographers. Bragg the younger describes a simple formula for the principle that determines the direction of the bent X-rays and shows that it is in full agreement with the theory by von Laue. The diffraction of X-rays can be described as specular reflection by a set of parallel planes through all lattice elements. A diffracted beam is obtained if

$$
2 d \sin \theta=n \lambda,
$$

where $d$ is the distance between the planes, $\theta$ is the angle of incidence, $n$ is an integer and $\lambda$ is the wavelength. Through this simple approach the determination of crystal structures was made possible. In the first step the zincblende diffraction pattern by von Laue was correctly interpreted by W. L. Bragg as a face-centred atomic arrangement irradiated by a continuous spectrum. This also led W. H. Bragg to convert his X-ray spectrometer for a more accurate approach to structure determinations (W. H. Bragg, 1913a).

Continuing his report, Gullstrand feels he has to educate his readers in basic crystallography and space geometry to be able to describe the crystallographic analysis of the alkali metal halides. It becomes evident from the work of W. L. Bragg that the crystals are not built of molecules of these compounds, but rather of the metal and halide ions forming separate lattices in which one type of ion is surrounded by six ions of the other type, all at the same distance. The determination of a number of more complex structures, including diamond and quartz, is also described.

Gullstrand also discusses the only known critical claim at the time, by William Barlow (1914). He felt that the alkali halides must be treated as molecules. Gullstrand finds his discussion to be incorrect and comments on the lack of response from Bragg as a kindness to Professor Pope, with whom Barlow was a co-worker.

The report also describes the work by W. H. Bragg on the development of the X-ray spectrometer and his studies of the relationship between the X-ray wavelengths, originating from different metal targets, and the absorption coefficients of different materials (W. H. Bragg, 1914a). He also studied the effect on the diffraction pattern due to change in temperature, which he found to agree with the theory by Debye (W. H. Bragg, 1914b).

Gullstrand then tried to evaluate the individual contributions by the two Braggs. He firstly concludes that in the determination of crystal structures W. L. Bragg has been the leading figure. However, the father had developed the equipment and investigated the properties of X-ray spectra from different metals. He was probably also the one who carried out most of the measurements. The contributions therefore seemed inseparable and any other outcome than a shared prize would be impossible. With the advent of the methods developed by the two Braggs, a whole new world had been opened up and already partly explored. However impressive the significance of these methods may seem, their potential can hardly be fully anticipated. To reward the discoverers of the new methods, after the discoverer of the new means, Max von Laue, has been rewarded, will create full justice. Thus the prize should be shared between W. H. Bragg and his son W. L. Bragg.

In their summary for the year (18 September 1915) the Nobel Committee for Physics discussed the analysis of X-ray spectra by Moseley and Darwin and considered their work to be more chemistry than physics. Thomas Alva Edison was nominated for wireless telegraphy. The committee concluded that such a prize would honour a lifetime of achievements, which was against the rules. This was also considered to be the case for Augusto Righi (Bologna). Max Planck (Berlin) was nominated for the quantum theory, but the committee found that they needed further clarification. Walter Nernst (Göttingen) was nominated for the heat theorem and Otto Lehmann (Karlsruhe) for liquid crystals. The two latter nominations were considered not to be competitive enough. Charles Thomson Rees Wilson (Cambridge) was nominated for his method of making the paths of electrically charged particles visible by condensation of vapour. Fredrik Carl Mülertz Størmer (Christiania) was nominated for the insights into the movements of electric particles close to a magnet and for his studies of the Northern Lights. Aime Cotton (Paris) was nominated for his work on optics and dichroism, and 
Louis Carl Heinrich Friedrich Paschen (Tübingen) for blackbody radiation. George Ellery Hale (Carnegie Observatory) was also nominated. In addition, one of the nominations concerned a very much appreciated way of coupling railway cars.

In their conclusion, the committee went entirely along with the suggestion by Gullstrand in recommending that the Academy award Max von Laue the physics prize for 1915, in the case that he had not already obtained the prize for 1914. If

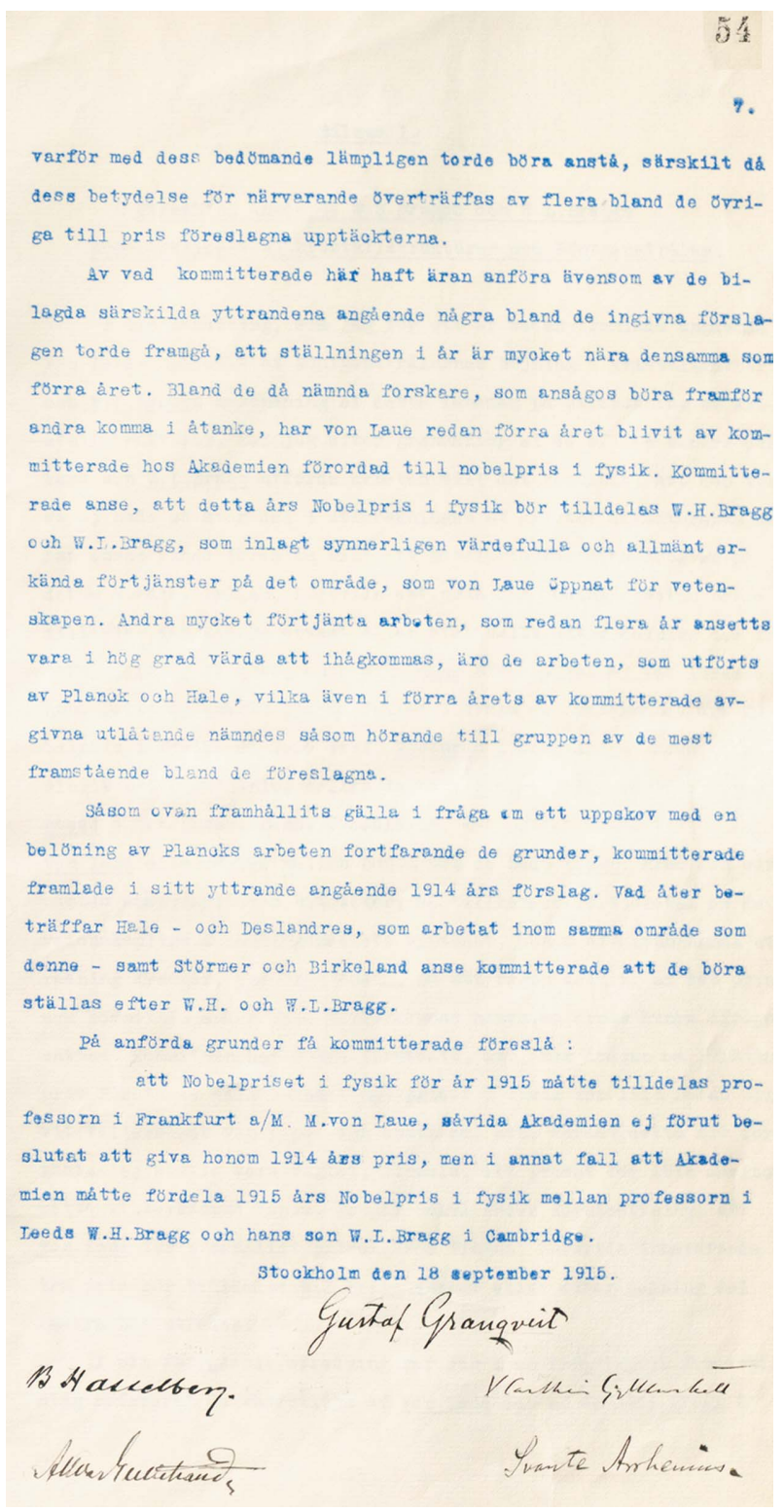

Figure 1

The last page of the summary by the Nobel Prize Committee for Physics dated 18 September 1915 with the signatures of its five members. They concluded by recommending that the Academy should award Max von Laue the Nobel Prize in Physics for 1915, if he had not already been awarded the prize for 1914. If von Laue had already been awarded the prize for 1914, the prize for 1915 should be shared between Professor W. H. Bragg, Leeds, and his son W. L. Bragg, Cambridge. Reproduced with the permission of the Centre for History of Science, The Royal Swedish Academy of Sciences. von Laue was already awarded the prize for 1914 then the two Braggs should be given the prize for 1915 (Fig. 1).

At the Royal Academy meeting on 11 November 1915 (present: President Eriksson, Secretary Aurivillius and 82 additional members) it was announced that the Academy was not permitted to delay the awarding of the Nobel Prizes for 1914 and 1915 until 1916. Thus, the documents from the Nobel Committees for 1914 and 1915 should be discussed to reach decisions on the award of the prizes for both these years. It was then concluded that Professor Max von Laue, University of Frankfurt, was to obtain the prize for physics for 1914 'for his discovery of the diffraction of X-rays by crystals'.

The Academy met again the next day (12 November 1915) to discuss the Nobel Prize in Physics for 1915. In addition to the President and Secretary 78 members were present. It was decided that Professor W. H. Bragg, University of Leeds, and his son W. L. Bragg, Cambridge, would be awarded the prize jointly 'for their services in the analysis of crystal structure by the means of X-rays'. The news was rapidly communicated to the recipients by telegram.

In a responding telegram to the Royal Academy on 15 November 1915, W. H. Bragg gratefully acknowledged the information that he and his son were to receive the Nobel Prize in Physics for the year (Fig. 2).

However, the war meant that the Nobel lectures and the prize ceremonies could not be held in the regular way. A ceremony for all laureates from during the war was delayed until 1920. Both W. H. and W. L. Bragg excused themselves from being able to come. This may illustrate the strong connections between science and politics during this period (Widmalm, 1995).

Before the First World War, Germany was a leading scientific nation. With the war national tensions grew drama-

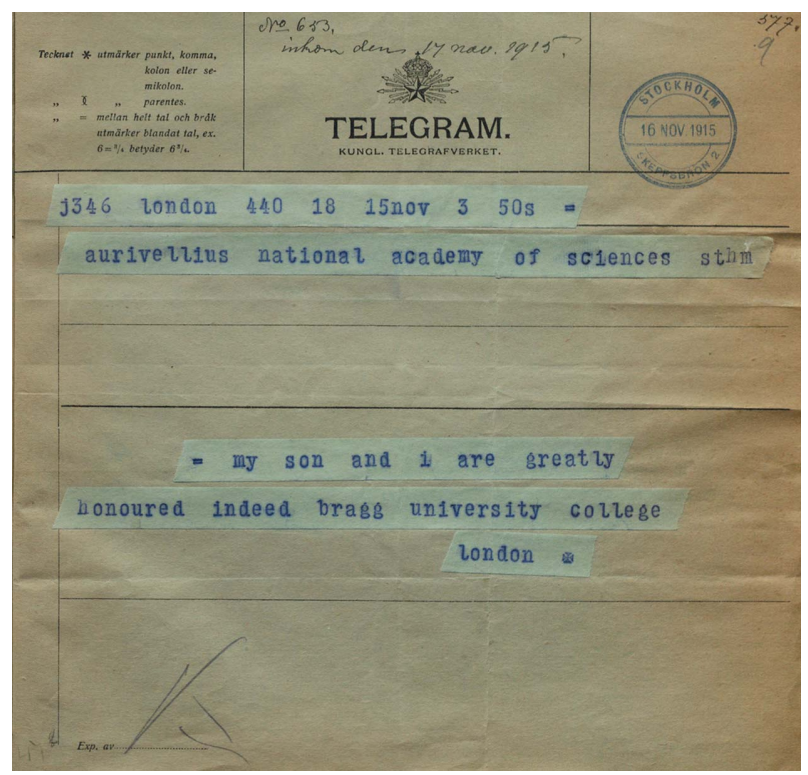

Figure 2

The telegram from W. H. Bragg acknowledging the message about the Nobel Prize in Physics, 1915. Reproduced with the permission of the Centre for History of Science, The Royal Swedish Academy of Sciences. 


\section{Bragg centennial}

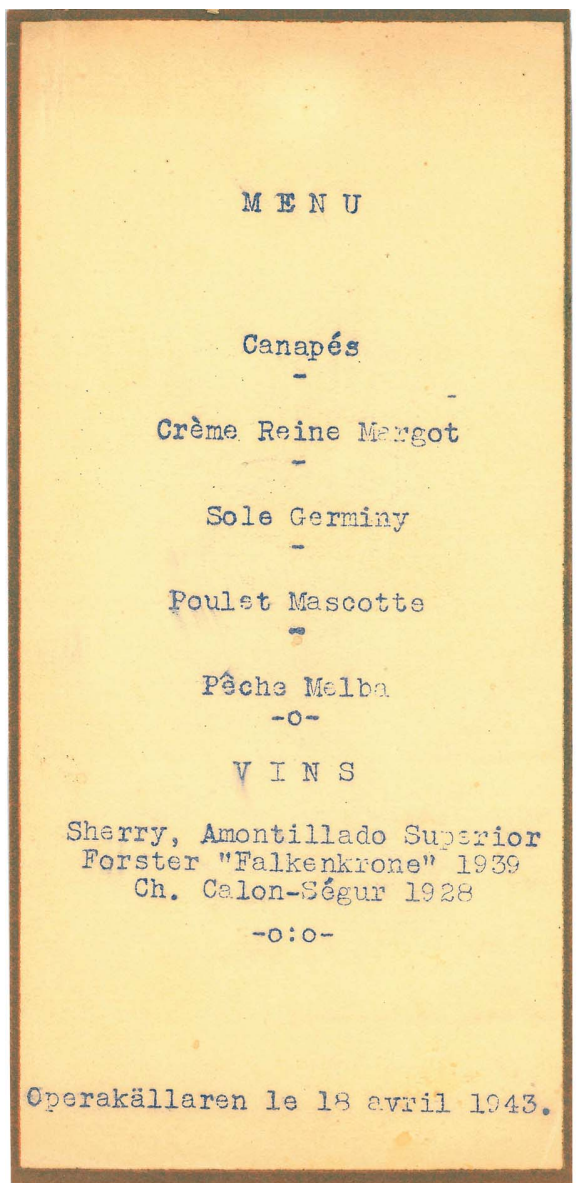

$(a)$

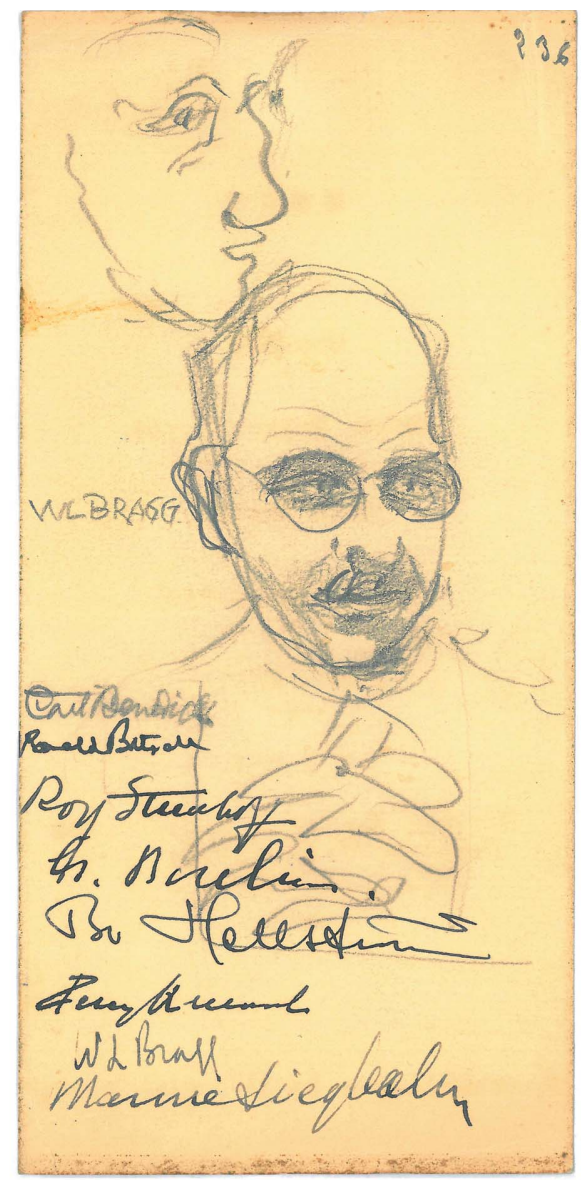

(b)

Figure 3

A drawing by Professor Carl Axel Fredrik Benedicks of W. L. Bragg on the reverse side of the menu from a dinner at the Opera cellar in Stockholm, 22 April 1943. The dinner participants signed the drawing. Reproduced with the permission of the Centre for History of Science, The Royal Swedish Academy of Sciences.

tically. Several German scientists who were awarded prizes during the war would participate in the ceremony. The absence of the Braggs could be due to nationalistic sentiments (Jenkin, 2008). In the case of W. L. Bragg it was agreed in communications with Svante Arrhenius that he would come to

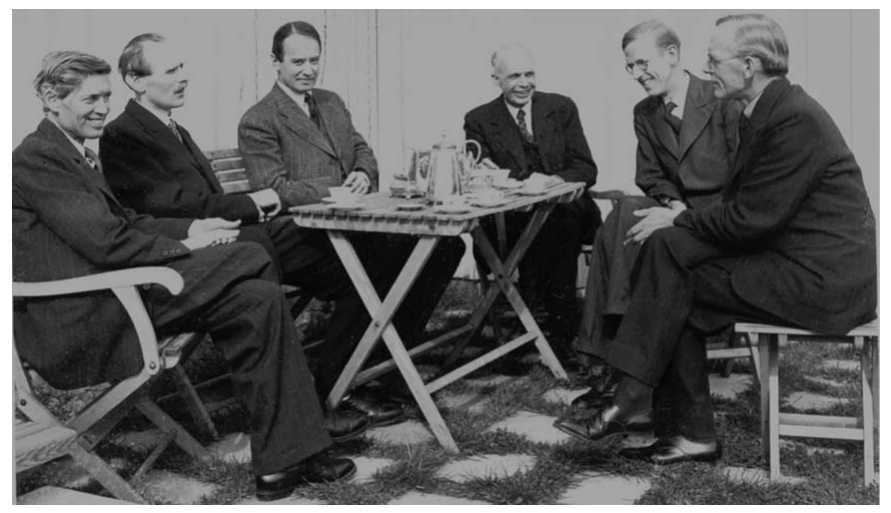

\section{Figure 4}

A gathering with the chemistry professors at Uppsala University, 22 April 1943. The Svedberg (Nobel laureate in chemistry, 1926), Arne Fredga, Arne Tiselius (Nobel laureate in chemistry, 1948), Lawrence Bragg, Gunnar Hägg and Axel Lindh.

present his Nobel lecture in September 1922 (W. L. Bragg, 1915). W. H. Bragg never gave a Nobel lecture (Jenkin, 2008).

Sir William Lawrence Bragg again visited Sweden, this time in April and May 1943, in the midst of the Second World War (Phillips, 1990). He flew to Stockholm on 16 April. The British Council arranged the trip (British Council, annual report, 1943-1944). This organization was established to promote a wider appreciation of British culture abroad to combat German and Italian cultural propaganda. Among its activities was the organization of lecture tours abroad by established British scientists. Sir William Henry Bragg founded the Council's Science Advisory Committee, of which he was chairman from 1940 until his death in 1942.

Much of Europe was inaccessible to the British Council during the war, either because of active conflict or German occupation. Sweden was one of only four European countries (the others were Portugal, Spain and Iceland) in which the Council could operate. An office in Stockholm was opened in 1941, which offered support to anglophile societies across the country. Lecturers were sometimes sent over from London, although transport to Sweden was frequently disrupted.

During the visit to Sweden in 1943, W. L. Bragg gave 14 lectures. He visited four universities and spoke on X-ray diffraction and in addition gave lectures at several anglophile societies. His ability to interpret science for the benefit of laymen made him a most popular visiting lecturer. During the

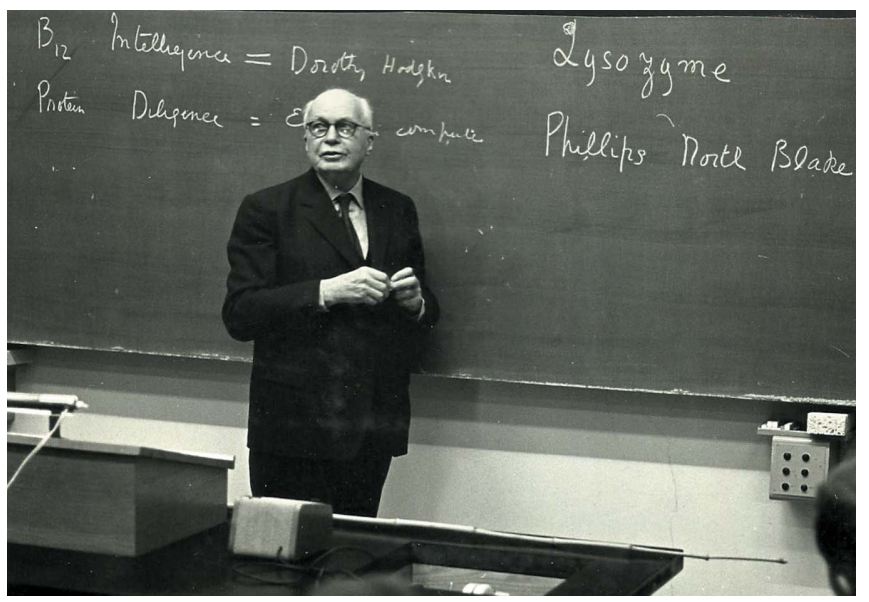

Figure 5

W. L. Bragg presenting the Nobel jubilee lecture in Uppsala, 1965. 
visit to Stockholm a dinner was held at the Opera cellar by a group of scientists, among them Manne Siegbahn, Nobel laureate in physics in 1924. Professor Benedicks frequently used his skills at making drawings of people in his company. At this occasion he made a sketch of W. L. Bragg on the reverse side of the menu (Fig. 3). The participants, including Bragg, signed the drawing.

During the visit to Uppsala on the same trip the chemistry professors all enjoyed the visit by their famous colleague, probably one of few visitors during the war (Fig. 4).

W. L. Bragg returned to Sweden on at least two more occasions. One was for the IUCr congress in 1951 and subsequently to celebrate the 50th anniversary of his Nobel Prize, 1965. During the last occasion he gave lectures at several universities (Fig. 5). He described the developments of X-ray crystallography aimed at solving an ever-increasing complexity of crystal structures. He had the fortune to bring along a model of the first enzyme structure, hen egg-white lysozyme, that had just been determined in his laboratory by David Phillips and colleagues.

I received valuable assistance in my search for documents in the Nobel Archives by Maria Asp Dahlbäck. I also received kind assistance with the photographs by Professors Ivar Olovsson and Bror Strandberg. Joanna Loxton at The British Council, London has provided details about the wartime visit to Sweden by W. L. Bragg. Professor Steve Wilkins provided a very valuable check of the content and presentation.

\section{References}

Arrhenius, S. (1915). Nomination to the Nobel Committees of Chemistry and Physics. January 30.
Barlow, W. (1914). Proc. R. Soc. London Ser. A, 91, 1-16.

Bragg, W. H. (1912). Nature (London), 90, 219.

Bragg, W. H. (1913a). Nature (London), 90, 572.

Bragg, W. H. (1913b). Nature (London), 91, 477.

Bragg, W. H. (1914a). Proc. R. Soc. London Ser. A, 89, 430-438.

Bragg, W. H. (1914b). Philos. Mag. 27, 881-899.

Bragg, W. H. \& Bragg, W. L. (1915). X-rays and Crystal Structure. London: Bell and Sons.

Bragg, W. L. (1913a). Proc. Cambridge Philos. Soc. 17, $43-57$.

Bragg, W. L. (1913b). Proc. R. Soc. London Ser. A, 89, 248-277.

Bragg, W. L. (1915). The diffraction of X-rays by crystals. Nobel lecture, September 6, 1922. http://www.nobelprize.org/nobel_ prizes/physics/laureates/1915/wl-bragg-lecture.html.

British Council (1943-1944). Annual Report, pp. 35, 117.

Eckert, M. (2012). Acta Cryst. A68, 30-39.

Friedrich, W., Knipping, P. \& Laue, M. (1912). Sitzungsber. Bayerische Akad. Wiss. 42, 303-322.

Gullstrand, A. (1914). Special report, July 3, 1914. Nobel Archive, Stockholm.

Gullstrand, A. (1915). Special report, June 25, 1915. Nobel Archive, Stockholm.

Jenkin, J. (2008). William and Lawrence Bragg, Father and Son. Oxford University Press.

Laue, M. von (1912). Sitzungsber. Bayerische Akad. Wiss. 42, $363-$ 373.

Nobel Committee for Physics. Report to the Royal Academy September 15, 1914. Nobel Archive, Stockholm.

Nobel Committee for Physics. Report to the Royal Academy September 18, 1915. Nobel Archive, Stockholm.

Phillips, D. (1990). Selections and Reflections: the Legacy of Sir Lawrence Bragg, edited by J. M. Thomas \& Sir D. Phillips, pp. 1-69. The Royal Institution of Great Britain.

Royal Academy of Sciences, Stockholm. Protocol, October 16, 1914. Nobel Archive.

Royal Academy of Sciences, Stockholm. Protocol, October 28, 1914. Nobel Archive.

Royal Academy of Sciences, Stockholm. Protocol of the Physics class. October 31, 1914. Nobel Archive.

Widmalm, S. (1995). Minerva, 33, 339-360. 